\title{
Sonrisas de esperanza: un proyecto de inclusión social
}

\author{
Smiles of hope, a project of social inclusion
}

\author{
Autoras \\ Luz Amarilis Martin Moya. Universidad San Gregorio de Portoviejo. Manabí. Ecuador. \\ lamartin@sangregorio.educ.ec.https://orcid.org/0000-0002-4095-439X \\ Addys Parra Cruz. Universidad San Gregorio de Portoviejo. Manabi. Ecuador. \\ aparra@sangregorio.edu.ec.http//orcid.org/0000-0002-8285-1946
}

Sandra Susana Parra Iraola. Universidad San Gregorio de Portoviejo. Manabí. Ecuador. ssparra@sangregorio.edu.ec

Celia Ponce Faula. Universidad San Gregorio de Portoviejo. Manabi. Ecuador. cfponce@sangegorio.educ.ec https://orcid.org/0000-0002-5117-3900.

Fecha de recibido: 2018-09-07

Fecha de aceptado para publicación: 2019-08-14

Fecha de publicación: 2018-09-30

\section{Resumen}

Los pacientes con algún tipo de discapacidad, tienen menor acceso a la atención odontológica, y requieren de sus cuidadores y de la gestión comunitaria para recibir estos servicios. Se realizó un corte transversal a los resultados del proyecto de Vinculación "Sonrisas de Esperanza" en la Carrera Odontología de la USGP, con el objetivo de caracterizar la población según variables demográficas, precisar el tipo de discapacidad, diagnóstico odontológico y tratamiento. El universo de estudio estuvo constituido por pacientes pertenecientes a la fundación FADINNAF, y sus cuidadores. La fuente primaria de la investigación fueron las historias clínicas, realizadas por los estudiantes y docentes. Las variables estudiadas fueron: edad, sexo, tipo de discapacidad, diagnóstico odontológico y tratamiento. Como principales resultados, se encontró que las discapacidades físicas fueron más frecuentes, predominó el sexo femenino, y el grupo etáreo de 50 a 59 años. Existe alto índice de caries, mala higiene bucal y dientes perdidos en la población estudiada; las actividades preventivas tuvieron mayor frecuencia en el estudio.

Palabras clave: Atención odontológica, caries, discapacidad, exclusión sanitaria del discapacitado índice de placa dentobacteriana.

\begin{abstract}
Patients with some type of disability have less access to dental care, and require their caregivers and community management to receive these services. A transversal cut was made to the results of the project of Linking "Smiles of Hope" in the Dentistry Career of the USGP, with the objective of characterizing the population according to demographic variables, to specify the type of disability, dental diagnosis and treatment. The universe of study consisted of patients belonging to the FADINNAF foundation, and its caregivers. The primary source of the research was the medical records, made by students and teachers. The variables studied were: age, sex, type of disability, dental diagnosis and treatment. As main results, it was found that physical disabilities were more
\end{abstract}


frequent, the female sex predominated, and the age group from 50 to 59 years. There is a high rate of caries, poor oral hygiene and missing teeth in the studied population; preventive activities were more frequent in the study.

Keywords: Dental care, caries, disability, health exclusion of the disabled dental plaque index.

\section{Introducción}

La Universidad San Gregorio de Portoviejo, una institución comprometida con la sociedad manabita, a través del proyecto "Sonrisas de Esperanza" contribuye a la salud oral de los discapacitados que se atienden en la fundación FADINNAF, pacientes con limitados recursos económicos e imposibilidad de realizarse tratamientos odontológicos de calidad; excluidos socialmente.

El proyecto Constituye un mecanismo y espacio estratégico de articulación y coordinación interinstitucional y multisectorial, para el diagnóstico, prevención, tratamiento y rehabilitación de enfermedades bucodentales de esta forma coexisten las 3 funciones sustantivas de la Universidad: Investigación, Docencia, y Vinculación.

La OMS (2011) define la discapacidad como "un fenómeno complejo que refleja una interacción entre las características del organismo humano y las características de la sociedad en la que vive”. En el 2013, a nivel mundial más de 1000 millones de personas convivían con alguna discapacidad, lo cual representó el 15\% de la población. Se estima que 190 millones de personas tienen dificultad para vivir normalmente y la tendencia va en aumento conforme se avanza en edad y aumentan las enfermedades crónicas (OMS, 2018).

El paciente discapacitado es aquel que independientemente de la edad, tendrá al examen físico, algún signo o síntoma que lo hacen diferente, pudiera tratarse de una discapacidad intelectual, física, o visual (Tan \& Rodríguez, 2002).

El manejo odontológico en pacientes en condición de discapacidad debe basarse en un sistema de estrategias comunicativas, protocolo de atención integrales, y multidisciplinarios, y deben incluir a los cuidadores en los procesos de diagnóstico y prevención. Y que se den a conocer oportunamente al estudiante de odontología del cuidado bucal dentro de su proceso de formación (Giraldo, Martinez, Cardona, Gutierres, Giraldo, Jimenez, 2017).

El cuidado de la salud Oral, en pacientes con discapacidad, o necesidades especiales, constituye un verdadero reto en el accionar de los odontólogos, en los pacientes donde coexisten discapacidad física e intelectual, no tienen un buen desarrollo cognitivo, no son capaces de comprender la importancia de la higiene bucal, además si hay asociada una discapacidad física, el proceso de remover la placa dentobacteriana con el cepillo, será muy difícil para él; será entonces la responsabilidad del cuidador. El cuidado inadecuado de la cavidad bucal, aumenta la incidencia y la prevalencia de caries y enfermedad periodontal (Morales, 2012).

El proyecto de Vinculación Sonrisas de Esperanzas, responde a las necesidades sociales, siempre crecientes, y enmarca a la carrera de odontología, en las tendencias 
actuales de la enseñanza universitaria, donde la investigación y la vinculación con la colectividad puntúan dentro de estándares de excelencia. Coincidimos con el Proyecto de la Universidad Ohio donde los estudiantes de odontología brindad atención a la comunidad, el programa beneficia tanto a pacientes como a los estudiantes de odontología que adquieren habilidades y competencias en la atención del paciente en una variedad de entornos. Los estudiantes adquieren experiencia enriquecedora una mejor comprensión de las opciones de carrera y una mayor conciencia social (Ohio University, 2019).

La investigación realizada tuvo como objetivo, caracterizar la población según variables demográficas; describir la población según tipo de discapacidad, diagnóstico odontológico y, realizar tratamientos odontológicos curativos a los beneficiarios de la fundación FADINNAF; además promover salud bucal en pacientes y cuidadores.

\section{Metodología}

Se realizó un estudio descriptivo transversal en la Carrera Odontología de la USG de Portoviejo, en el mes de marzo del año 2018. Con un universo de estudio $\mathrm{N}=300$ pacientes, de los cuales: 180 son pacientes con algún tipo de discapacidad y 120 son cuidadores. Hasta este momento en que se realiza la evaluación de los resultados del proyecto, han sido atendidos 150 pacientes, quienes conforman la muestra del actual estudio (n).

La población está localizada en Portoviejo en diferentes sectores de la ciudad: El Guabito, El Florón, Lea, Las Orquídeas (Comunidades urbanas), en Rio Chico y Francisco Pacheco (Comunidad Rural).

La revisión de las historias clínicas constituyó la fuente primaria de información.

Variables: edad, sexo, tipo de discapacidad, diagnóstico odontológico y tratamiento.

Los resultados fueron procesados en Microsoft Excel, se contrastan con literatura actualizada, y se expresan de forma textual y en tablas, de porciento y frecuencia.

\section{Resultados}

La Fundación FADINNAF, incluye 300 personas, de los cuales se diagnosticaron y atendieron 150 pacientes hasta el mes de marzo del 2018, lo que representa el 50\% de los beneficiarios. De ellos, 111 presentan algún tipo de discapacidad, y 39 son cuidadores. En los que se expresaron las siguientes variables demográficas. El grupo etáreo de mayor representatividad fue, de 50 a 59 años, predominó el sexo femenino con 92 pacientes para un 61\%. (Ver Tabla 1 en Anexos).

La discapacidad más frecuente en nuestra casuística fue, la física con el 54,95\% prevaleciendo el sexo masculino con 34 pacientes. (Ver Tabla 2 en Anexos)

Los investigadores, aplicaron a los 150 pacientes el Índice de Caries en dientes Permanentes 
(CPO) lo cual expresa el siguiente resultado. Se diagnosticaron con caries 83 pacientes. El promedio de piezas cariadas, perdidos por caries y obturados (CPO), fue de $3,7 \%$ es decir hay 556 piezas afectadas por caries, piezas pérdidas por caries $6,26 \% ; 940$ piezas dentales y piezas obturadas $1,04 \%$ para 167 piezas dentales.

Si bien los pacientes pediátricos no fue el grupo de mayor prevalencia, es válido señalar que también en ellos se midió el índice de caries en dientes temporales (cop). El promedio de piezas cariadas en niños es 0,18 es decir 28 dientes afectados por caries, observando en el cuadro que no hay piezas extraídas y obturadas.

La higiene bucal, es un parámetro vital, cuando de Salud Bucal se trata, por ello, los investigadores examinaron cuidadosamente este parámetro, obteniendo como resultado que el promedio de placa es de 0,68 , el de cálculo 0,36 dando un promedio del grupo estudiado de 1,047 de HIOS.

A los pacientes diagnosticados se les realizaron tratamientos odontológicos como restauraciones (clase I, II, III, IV, V) para un total de 30 restauraciones realizadas, lo que representa el $26 \%$ de los tratamientos. La profilaxis y fluorizaciones fueron aplicadas en 84 pacientes, para un $74 \%$.

La promoción de salud bucal fue brindada en cada uno de los escenarios donde los integrantes del proyecto de vinculación brindaron sus servicios.

\section{Discusión}

Los pacientes con diferentes necesidades especiales representan un desafío para los odontólogos, el profesional de salud accionará en las enfermedades bucales prevenibles de origen dentobacteriana y junto a los cuidadores, minimizará el impacto de las principales necesidades de las personas en condición de discapacidad con respecto a su higiene oral (Marulanda, Betancur, Espinosa, Gómez \& Tapias, 2014).

Los cuidadores, muchas veces descuidan su salud y como resultado se afecta la salud bucal, estudios como el realizado por (Freire da Silva, Müller, Drawanz, Rocha, \& Sousa, 2018) identifican la necesidad de que los cuidadores deben preocuparse por este particular, y acudir a centros de atención odontológica.

En el estudio se encontró que las discapacidades intelectuales no fueron las más distintivas, pero, si se evidencia que en los pacientes con retardo mental moderado o severo, y en los que están diagnosticados con Sindrome de Dawn la higiene bucal es pobre, lo que incide en la aparición de caries y enfermedades periodontales, nuestro estudio, al realizar el índice de caries, en dientes permanentes y temporales, coincide con otros investigadores los plantean que "Dos subgrupos con un riesgo especialmente alto para los problemas de salud oral son las personas con síndrome de Down y las personas que no pueden cooperar para la atención dental de rutina” (Anders, Davis, 2010).

Lo corroborado en la investigación actual, y también por la literatura, permite, afirmar que la salud bucal de los discapacitados es deficiente si se compara con la población normal, pues existe una prevalencia universal de la enfermedad periodontal y una higiene bucal más pobre. La alta incidencia de dientes cariados no tratados y la mayor incidencia de dientes ausentes (Waldmana, Permans, 2002). 
"La profilaxis dental profesional se realiza para eliminar las placas depositadas en la superficie de los dientes o en la zona subgingival para tratar o prevenir la caries dental y la enfermedad periodontal" (Axelsson, Lindhe, Wäseby, 1976). En los casos estudiados la mayor parte de los tratamientos se encaminaron en un mayor porcentaje a realizar la profilaxis.

La utilización efectiva de fluoruros tópicos en la prevención de caries, dependerá del nivel de conocimiento del profesional sobre el uso apropiado de este; los estudiantes en la vinculación, aplican estas terapias a sus pacientes, y reafirman sus conocimientos sobre el tema. "La decisión sobre los tratamientos preventivos, como los fluoruros tópicos, está influenciada por los conocimientos adquiridos durante el entrenamiento de pregrado" (Muralidharan, Pocha, \& Paul, 2018).

Es valido señalar que en este proyecto juegan un papel importante, los lideres de la fundación FADINAF, quienes se convierten en facilitadores en las labores de atención odontológica, estudios publicados, reseñan lo importante de la comunidad en las actividades de promoción de salud. (Mathu-Muju, Kong, Brancato, McLeod \& Bush.H.M, 2018).

La actitud del odontólogo, frente a un paciente con discapacidad es de gran valor, coincidimos con el estudio realizado por Odontóloga María Alejandra Quintero Salazar Especialista en Odontopediatría y colaboradores, en que existe aceptación por parte del profesional de odontología frente a la discapacidad, en su trabajo, también se pronuncian sobre la necesidad de que las políticas de estado se involucren referente al conocimiento de la existencia de estos pacientes y sus necesidades especiales, para que sean incluidos en los diferentes programas de salud, lo que elevará la calidad de vida de estos sectores poblacionales vulnerables (Salazar, Padrón \& Salazar,2005).

Las personas con discapacidades tienen menos acceso a la atención médica odontológica, en comparación con las personas sin esta afección. Las politicas de salud, de diferentes paises, dejan, lastimosamente en exclución, a aquellos que presentan algun grado de discapacidad intelectual y del desarrollo (Havercamp \& Scott, 2015 ). Es por ello que el proyecto de Vinculación Sonrisa de Esperanza se convierte desde sus inicios en un espacio de inclusión para etas personas que por su condición han sido marginadas. Nuestro proposito es brindarles atención odontológica con calidad y calidéz, poniendo en alto la misión social de La Universidad San Gregorio de Portoviejo.

\section{Conclusiones}

Las discapacidades físicas fueron las más prevalentes en nuestra casuística.

Predominando el sexo femenino, y el grupo etáreo de 50 a 59 años.

Existe Alto índice de caries, mala higiene bucal y dientes perdidos en la población estudiada.

Las actividades preventivas tuvieron mayor representatividad en el estudio.

El proyecto Sonrisas de Esperanzas, logró la inclusión y atención odontológica con calidad y calidez, en pacientes y cuidadores pertenecientes a la fundación FADINAF. 


\section{Referencias Bibliográficas}

Anders, P. L., \& Davis, E. L. (2010). Oral health of patients with intellectual disabilities: a systematic review. Special Care in Dentistry, 30(3), 110-117.

Axelsson, P., Lindhe, J., \& Wäseby, J. (1976). The effect of various plaque control measures on gingivitis and caries in schoolchildren. Community dentistry and oral epidemiology, 4(6), 232-239.

da Silva Júnior, I. F., Stüermer, V. M., Hartwig, A. D., Scharsdosim, L. R., \& Azevedo, M. S. (2018). Use of dental care services and related factors in students with special health care needs in a rehabilitation and education center. $R S B O, 15(1), 5-11$.

Giraldo-Zuluaga MC, Martínez Delgado CM, Cardona-Gómez N, Gutiérrez-Pineda JL, GiraldoMoncada KA, Jiménez-Ruíz PM. Manejo de la salud bucal en discapacitados. Artículo de revisión. Rev. CES Odont 2017; 30(2): 23-36.

Havercamp, S. M., \& Scott, H. M. (2015). National health surveillance of adults with disabilities, adults with intellectual and developmental disabilities, and adults with no disabilities. Disability and Health Journal, 8(2), 165-172.

Mathu-Muju, K. R., Kong, X., Brancato, C., McLeod, J., \& Bush, H. M. (2018). Utilization of community health workers in Canada's Children's Oral Health Initiative for indigenous communities. Community dentistry and oral epidemiology, 46(2), 185-193.

Marulanda, J., Betancur, J. D., Espinosa, S., Gómez , J. L., \& Tapias, A. (2014). Salud oral en discapacitados. Red Iberoamericana de Expertos en la Convención de los derechos de las personas con discapaciadad.Recuperdo en http://repositoriocdpd.net:8080/handle/123456789/119

Morales, C., \& Mariana, C. (2012). Atención odontológica a pacientes especiales: una realidad creciente. Acta Odontol Venez, 50(1).

Muralidharan, D., Pocha, S., \& Paul, A. (2018). Topical fluoride use: Knowledge, attitudes and practices of undergraduate dental students of three Dental Colleges in South India. European Journal of Dental Education, 22(3), e444-e450

Ohio University. (16 de Mayo de 2019). OSU.EDU. Obtenido de https://dentistry.osu.edu/aboutus/community-outreach-education/oral-health-improvement-through-outreach-ohio-project

OMS.(2011). Informe mundial sobre discapacidad. OMS. Recuperado en https://www.who.int/disabilities/world_report/2011/es/

OMS. (15 de marzo de 2018). Organización Mundial de la Salud. Obtenido de Organización Mundial de la Salud: http://www.who.int/topics/disabilities/es/

Salazar, C., Padrón, M., \& Salazar, J. L. (2005). Actitud del odontólogo hacia los niños y adolescentes discapacitados. Acta odontológica venezolana, 43(3), 240-251.

Tan Castañeda, N. \& Rodríguez Calzadilla, A. Correspondencia entre la formación académica del estomatólog relacionado con pacientes especiales y la práctica estomatológica integral. Rev. Cuba. Estomatol.,38(3):181-91, 2001

Waldman, H. B., \& Perlman, S. P. (2002). Why is providing dental care to people with mental retardation and other developmental disabilities such a low priority? Public Health Reports, 117(5), 435. 


\section{Anexos}

Tabla 1: Distribución de Pacientes con Discapacidad y cuidadores según Sexo

$\begin{array}{lrccc}\text { Sexo } & \text { Paciente } & \text { Cuidador } & \text { Total } & \% \\ \text { Femenino } & 69 & 23 & 92 & 61,33 \\ \text { Masculino } & 42 & 16 & 58 & 38,66 \\ \text { Total } & 111 & 39 & 150 & 100 \\ \% & 74 & 26 & 100 & 100\end{array}$

Fuente: Historias Clínicas

Tabla 2: Distribución según sexo y el tipo de discapacidad.

\begin{tabular}{lcccccc} 
Discapacidad & Femenino & $\%$ & Masculino & $\%$ & Total & $\%$ \\
Físicos & 27 & 24,32 & 34 & 30,63 & 61 & 54,95 \\
Visuales & 15 & 13,51 & 1 & 0,9 & 16 & 14,41 \\
Auditivos & 12 & 10.81 & 3 & 2,7 & 15 & 13,51 \\
Intelectuales & 6 & 5,4 & 3 & 2,7 & 9 & 8,1 \\
Mentales & 9 & 8,1 & 1 & 0,9 & 10 & 9 \\
\multicolumn{1}{c}{ Total } & 69 & 60,36 & 42 & 37,83 & 111 & 100
\end{tabular}

Fuente: Historias Clínicas de los 111 pacientes con discapacidad. 